Disclosure: The author reports no disclosures. Go to Neurology.org/cp for full disclosures.

Correspondence to: gcascino@mayo.edu

1. Hauser WA, Annegers JF, Rocca WA. Descriptive epidemiology of epilepsy: contributions of populationbased studies from Rochester, Minnesota. Mayo Clinic Proc 1996;71:576-586.

2. Hauser WA, Rich SS, Lee JR, Annegers JF, Anderson VE. Risk of recurrent seizures after two unprovoked seizures. N Engl J Med 1998;338:429-434.

3. Berg AT. Risk of recurrence after a first unprovoked seizure. Epilepsia 2008;49(suppl 1):13-18.

4. Herman ST. Single unprovoked seizures. Curr Treat Options Neurol 2004;6:243-255.

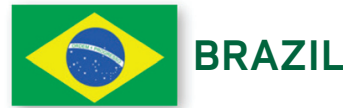

Andre Palmini, MD, PhD, Severe Epilepsies Outpatient Clinic \& Porto Alegre Epilepsy Surgery Program, Service of Neurology, Hospital São Lucas, and Faculty of Medicine and The Brain Institute (InsCer), Pontificia Universidade Católica do Rio Grande do Sul (PUCRS), Porto Alegre, Brazil: The information era has narrowed the "conceptual" gap as to how to evaluate patients with medical problems. Nonetheless, countries differ in critical mass of expert practitioners, accessibility to resources, and level of medical and general education. Therefore, particularly in large countries like Brazil, specific approaches relate to socioeconomic, geographic, and cultural constraints.

The evaluation of an unprovoked seizure in an adult is a good example. As is likely the case in most parts of the world, this person will be taken to an emergency room. There, the ideal scenario of careful history taking, paying attention to events or circumstances suggesting previous mild seizures, a psychogenic nonepileptic event, or a convulsive syncope, seldom happens. Thus, patients are usually managed as if that was a first epileptic attack, except in circumstances obviously related to acute alcohol withdrawal or overt abuse of illicit drugs. In many instances - even if the seizure has already stopped - that person will receive an IV or IM dose of an antiepileptic drug (AED), usually diazepam, phenytoin, or phenobarbital. Following routine procedures such as testing for blood glucose and electrolytes and a blood cell count, the patient will most likely be evaluated with a CT scan. If the latter does not indicate an acute lesion, then he or she will be referred to a neurologist in the community.

Approaches directed by a clinical neurologist can vary significantly according to regional prevalence of specific disorders and access to specialized professionals and facilities. If the CT was normal, the standard approach is to arrange for an EEG which, if also normal, will be considered in the decision either to withhold the introduction of an AED or to start medication irrespective of that result. The latter is usually the case when access to clinical monitoring services is more difficult, such as in distant rural areas or where the public health care system is less capable of providing for specialized care. When CT or EEG are abnormal, then medication is usually started. The presence of small cortical calcifications will be valued differently depending on whether the region is or is not endemic for cysticercosis, although the decision to withhold treatment in the presence of calcifications even in endemic areas is delicate.

The increased availability of MRI scanners is progressively modifying the standard approach. In many large and middle-sized cities around the country, an adult patient with an unprovoked seizure considered to be a first epileptic attack would have an MRI, which may show a small, unsuspected lesion, thus often clarifying the mechanism and the etiology of the seizure event. When MRI is also normal, the patient will follow the decision taken in conjunction with the specialist, namely, to start an AED or be followed without medication.

Improved education for patients and doctors is also likely to change the scenario. Patients are ever more uncomfortable with the lack of clear explanations for a significant event such as a seizure and will demand more from doctors. These, in turn, are becoming more aware of subtle clinical manifestations that could help explain the later occurrence of an unprovoked convulsive 
seizure, such as the presence of previous, recurrent, nonconvulsive, or mildly convulsive epileptic attacks (e.g., morning myoclonus), and also the differential diagnosis of an epileptic seizure.

The bottom line is that the potential for improvement of the approach to and care of a patient with an unprovoked seizure is unequivocal. The main goal is to move beyond the obvious scenarios and improve management of those situations in which a seizure occurs and all tests are normal. An unacceptably large proportion of these patients are either taking AEDs for years for nonepileptic disorders or having avoidable epileptic seizure recurrences because a diagnosis of epilepsy was not made.

Finally, there are a number of external modifiers to the standard overall approaches. For instance, recent data indicate that only $25 \%$ of Brazilians are covered by private health insurance and MRI scanners and EEG laboratories are less available to patients seen under coverage of the public health system, particularly outside major university hospitals. Thus, MRI and EEG will take longer in these patients, implying that coverage of the evaluation costs and differences in access to facilities in urban and rural areas do have an impact on management. This necessarily raises the issue of what would be the best approach, to begin with an AED even without a comprehensive workup or withhold the prescription until more information is available. Inevitably, these end up being individual decisions, but the trend is toward prescribing an AED to patients in these circumstances.

Ideally, patients with an unprovoked seizure should undergo detailed medical history and neurologic examination, routine blood tests, as well as an MRI scan and an EEG as soon as possible. When the likelihood of an epileptic mechanism is high on the list or the potential negative psychosocial impact of a second attack is thought to be significant, then the threshold to begin with an AED should be lowered. Because in Brazil AED costs are not reimbursed by private insurers and the public health system provides only a limited number of AED types, the medication started will vary according to socioeconomic and psychosocial profiles in different regions of the country. In many rural areas this translates into phenobarbital, while in other regions doctors may prescribe either valproate or a sodium channel blocker such as carbamazepine, oxcarbazepine, or phenytoin. Of course, considerations on the epileptic seizure type and special situations such as pregnancy should apply, although such choice is also on a learning curve.

Disclosures: Dr. Palmini reported serving as a board member and receiving payment for lectures and travel from Novartis, Abbott, Janssen-Cilag, and Eli Lilly; receiving payment for preparation of educational material from Novartis, Abbott, and Janssen-Cilag; and receiving payment for organization of preceptorships and workshops paid to fund fellowships from Novartis and Biogen. Go to Neurology.org/cp for full disclosures.

Correspondence to: apalmini@uol.com.br

\section{-7) INDIA}

Kurupath Radhakrishnan, MD, FAAN, Chaturbhuj Rathore, MD, Ramshekar Menon, MD, R. Madhavan Nayar Center for Comprehensive Epilepsy Care, Sree Chitra Tirunal Institute for Medical Sciences and Technology, Trivandrum, Kerala, India: There is no uniform approach to assessment of an unprovoked seizure in an adult in India. Due to the huge socioeconomic differences among its vast population and the varied levels of available health care, the approach differs significantly across the country. Certain unique features of health care in India have direct bearing on any such approach. First, most of the health care costs are directly borne by the patient or caregivers. Secondly, the majority of the population live in rural areas and are cared for by primary care physicians or internists who have little training in the evaluation and management of seizures and do not have access to perform or expertise to interpret EEG or CT scans. ${ }^{1}$ On the positive side, phenytoin and phenobarbital are provided free of cost to all patients at primary and secondary level state-run hospitals. Similarly, the charges for various investigations including EEG, CT scan, and MRI are subsidized in the state-run tertiary care centers according to the economic status of the patient. Prevalence of neurocysticercosis within a region also influences this approach. The authors have worked in different regions of the country at various levels within the health care system and have 